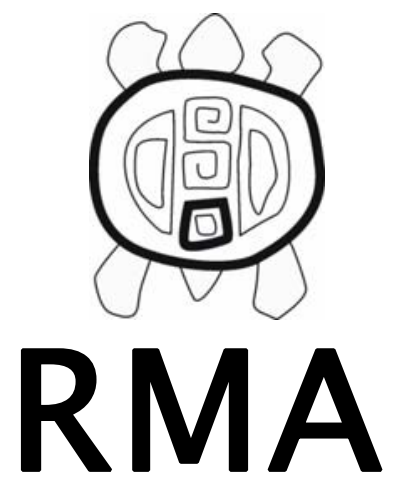

Arqueología

\title{
¿Cómo hacer chicha en Córdoba? Reflexiones en torno a los aspectos políticos y materiales de la producción
de chicha por inmigrantes bolivianos de chicha por inmigrantes bolivianos
de materiales de la producción
}

\author{
Francisco Pazzarelli \\ Gisela Vargas Ibarra² \\ ' CONICET / Museo de Antropología, FFyH, UNC. \\ fpazzarelli@hotmail.com \\ 2 Museo de Antropología, FFyH, UNC. \\ giselavargas06@hotmail.com
}

\begin{abstract}
Resumen
Las bebidas y las comidas pueden ser consideradas como instancias con una cotidianeidad particular, y con una poética y una gastro-política específicas, que se involucran en la reproducción cotidiana y en los sentidos de pertenencia de un grupo. En esas instancias convergen distintos sujetos $y$ materialidades que se articulan en un proceso en el que ellos mismos son definidos. Desde esta perspectiva, nos interesa reflexionar en torno de los procesos de producción de chicha (bebida fermentada a base de maíz) por parte de mujeres inmigrantes bolivianas y su articulación con otros contextos en la ciudad de Córdoba (Argentina). Consideramos que existe una tensión entre la necesidad de contar con esta bebida para ciertas celebraciones y la dificultad que existe para producirla, y que dicha tensión se inscribe en las situaciones de marginalidad que experimentan la mayoría de los inmigrantes. Para aproximarnos a la gastro-política que se despliega en estos procesos de producción, nos concentraremos en las trayectorias de las mujeres chicheras, así como de las materias primas y objetos necesarios para su elaboración.
\end{abstract}

Palabras clave: gastro-política, cultura material, chicha, migración boliviana, Córdoba

How to do chicha in Cordoba? Reflections on the political and material aspects of the production of chicha by Bolivian immigrants

\begin{abstract}
Beverages and food might be considered as instances with a special daily routine, and with a poetic and a special gastro-politic, which implicates in the everyday reproduction and in the sense of membership in a group. In these instances different subjects and materialities convergence, which articulates in a process in which themselves are defined. From this perspective, it interests us to reflect around the «chicha» (a fermented beverage based on maize) production processes from the view of the Bolivian women immigrants and it articulation with other contexts in the city of Córdoba (Argentina). We consider that it exists a tension between the necessity to count with this beverage for certain celebrations and the difficulty to produce it, and the tension which are inscribed in the marginal situations that experience the majority of the immigrants. To approximate the gastro-politics that unfolds in these production processes, we concentrate on the trajectories of the "chichera» women, as the source materials and other necessities for its elaboration.
\end{abstract}

Keywords: gastro-politics, material culture, chicha, Bolivian immigration, Córdoba

\section{Introducción: entre el hacer y el beber}

Las bebidas y las comidas son instancias que cuentan con una cotidianeidad particular, y poseen una poética y una gastro-política específicas (Appadurai 1981) que se involucran en la definición de espacios sociales y en la reproducción de la vida cotidiana. Con esta perspectiva, en este trabajo reflexionamos en torno de la producción de chicha (bebida fermentada a base de maíz) por parte de inmigrantes bolivianos, residentes en la ciudad de Córdoba, Argentina. Intentamos realizar una aproximación a los conflictos, tensiones y negociaciones que supone esta práctica en el marco de un contexto adverso, de 
marginalización y discriminación hacia los migrantes. Dentro de estas situaciones, también nos enfocaremos en la cultura material involucrada en estas prácticas, que definen un modo particular de hacer chicha en Córdoba.

Con estos intereses, incorporamos la categoría gastro-política, acuñada por Appadurai (1981), para considerar a los conflictos y negociaciones que surgen en torno a las transacciones de recursos alimenticios y económicos, en estrecha relación con la producción, presentación y consumo de comidas y bebidas. Para Appadurai (1981:494), las comidas (y las bebidas), consideradas como hechos sociales condensados, poseen una "virtuosidad semiótica» fundada en su cotidianeidad y en su involucramiento en la constitución de los discursos sociales diarios, que a su vez se imbrican en la reproducción de las relaciones sociales domésticas. Este potencial comunicativo y performativo de las comidas las hace objeto de disputa y negociación. Así, se despliegan estrategias gastro-políticas que, mediante la manipulación directa de la comida o de los contextos de su producción, presentación y consumo, tienen el interés de construir relaciones de simetría o asimetría, de reproducir jerarquías o de reforzar lazos parentales.

La introducción de esta categoría en el análisis de la producción de chicha en la ciudad de Córdoba encuentra sus razones en dos aspectos, que se implican mutuamente. Por un lado, la elaboración de esta bebida en el contexto cordobés se define por el conflicto permanente que sus productoras deben enfrentar en la obtención de la materia prima necesaria, así como del tiempo y de los objetos necesarios para llevar a cabo la producción; estos conflictos se inscriben en las condiciones económicas y materiales de marginalidad y subalternidad que la mayoría de los migrantes experimenta. Por otro lado, la producción de una bebida como la chicha, consumida en fiestas y celebraciones y con una importante carga simbólica y social, se involucra como un factor de importancia en la producción de «lo local» (Appadurai 2000) y de los sentidos de pertenencia de la comunidad inmigrante. En relación a los procesos migratorios, aquí podemos entender lo local como una «estructura de sentimientos» que se produce mediante distintas formas de actividades intencionales, que a su vez generan diferentes efectos materiales (Appadurai 2000:191), y que pone en relación a distintas escalas espaciales y temporales. Esta producción no supone que los sujetos repitan mecánicamente sus contextos nativos; implica, en cambio, una relación dialéctica en la que los mismos sujetos son producidos al tiempo que generan nuevos contextos (en sentido análogo a la relación entre estructuras y agentes estructurantes, Bourdieu y Wacquant 1995). Entre las técnicas de producción de lo local, pueden considerarse, por supuesto, a la elaboración y consumo de comidas y bebidas. Así, el análisis de la producción de chicha en Córdoba se enmarca en la tensión que existe entre las dos razones enunciadas: la dificultad de elaborar chicha y la importancia de contar con ella para ciertas celebraciones y fiestas; entre la dificultad de hacer y la importancia de beber se define la especificidad de su gastro-política.

En estas instancias convergen distintos tipos de agentes y de materialidades, en un proceso en donde todos ellos son definidos; la cultura material implicada en la elaboración de chicha ocupa un lugar importante en tanto puede considerarse inscripta en las técnicas de producción espacial -y material- de lo local (Appadurai 2000:189). Estas técnicas no deben ser analizadas como fines en sí mismos sino contemplando su «efectos más abstractos», que es la producción de lo local en tanto estructura de sentimientos (Appadurai 2000).

En un sentido similar, desde algunos planteos en arqueología se postula que los objetos no sólo cumplen con «funciones» sino que también se encuentran implicados de manera activa en la constitución de las prácticas sociales y contextos de los que toman parte (Shanks y Tilley 1987; Hodder 1990). Estos planteos encuentran su origen dentro del marco de la teoría social contemporánea, en la teoría de la estructuración (Giddens 1984) y en la teoría de la práctica (Bourdieu 1977). Así, la cultura material ya no es considerada como un simple medio para satisfacer necesidades (o, en nuestro caso, para hacer chicha). En cambio, es analizada en tanto se involucra en las prácticas de individuos posicionados (Bourdieu y Wacquant 1995), con los que entablan relaciones dialógicas en las que se construyen los significados de los contextos en los que interactúan.

Planteado el problema, consideramos que este trabajo puede aportar a las discusiones en torno de las prácticas de elaboración de bebidas y comidas, con su cultura material asociada, como implicadas en la producción de lo local. Por otro lado, y a través de la aproximación a la gastro-política de la producción de chicha, este trabajo se constituye como un modo de actualizar el reclamo acerca de las condiciones de marginalidad a la que se ven sometidos los migrantes en la ciudad de Córdoba.

\section{La chicha en el mundo andino y en Córdoba}

La chicha es una bebida realizada a base de la fermentación del maíz principalmente (aunque también de cebada, quínoa y maní), que se 
consume en todo el mundo andino desde momentos prehispánicos. Es un elemento infaltable en diferentes fiestas y celebraciones, tanto públicas como privadas, donde se encuentra condensando variados aspectos sociales y simbólicos de la estructuración de la vida cotidiana, así como de los sentidos acerca del pasado, presente y futuro. De todas formas, la chicha es una bebida que también se consume de manera cotidiana, aportando gran cantidad de nutrientes y constituyéndose en una fuente importante de alimento y de encuentro (Paredes 1936; Cutler y Cárdenas 1985 [1947]; Saignes 1993).

Tal como resume Saignes (1993), los estudios funcionalistas sobre consumo de alcohol en los Andes, que entienden a la borrachera como una manera de quitar las inhibiciones o sobrellevar una realidad conflictiva, son insuficientes para comprender todas sus dimensiones. En los Andes, el beber se encuentra relacionado con la reproducción de los ciclos vitales, con el diálogo constante con dioses y antepasados, y como espacio para el desafío del poder y lo establecido. De hecho, la presencia de la chicha (así como de otros líquidos fermentados) se registra como un elemento infaltable en distintos tipos de contextos rituales, ceremoniales y festivos (Arnold et al 1998; Fernández Juárez 1997). Abercrombie (1993:166-167) también señala la importancia de las challas y libaciones con alcohol en la conformación de los «caminos de la memoria» y en la constitución de «mecanismos poéticos» para la producción de los sujetos en contextos rituales.

Con respecto a la elaboración de chicha, se ha argumentado que a través de ese proceso se logra conferir un valor socializado a la bebida, transformando lo natural en cultural a través de la fermentación y de la acción de encerrar al líquido dentro de un artefacto, como puede ser una vasija (Saignes 1993:11). En un sentido similar, en el análisis de la política de la borrachera que realiza Randall (1993) se argumenta que en cada uno de los pasos de la elaboración de la chicha se re-produce el orden cósmico que estructura la vida simbólica de los pueblos.

Este proceso de elaboración de chicha implica mucho tiempo y diversidad de tareas, en las que se encuentran implicadas generalmente las mujeres ${ }^{1}$ chicheras. Las chicheras detentan un conocimiento que remite no sólo a una receta sino a un «saber-hacer» que se pone en práctica en cada proceso de producción. Para el mundo andino en general, se ha considerado de especial importancia que sean las mujeres aquellas que producen la chicha y las que, en determinadas circunstancias, se emborrachen para cantar y realizar libaciones en los momentos de siembra (Arnold et al.1998).
Suelen ser las encargadas, además, del cuidado de los objetos utilizados en el proceso de elaboración y de guardar las recetas. Se ha argumentado también que desde los tiempos de la colonia, y ante la desarticulación de antiguas redes sociales, el "oficio» de chichera se habría convertido en una forma de elevación social de las mujeres que residían en los poblados y que a partir de ese momento se ubicaron al frente de las chicherías (establecimientos para la venta de chicha) (Paredes 1936).

Aunque el consumo de chicha ha sido frecuentemente objeto de análisis por parte de la antropología, y se ha señalando la importancia que adquiere en distintos contextos rituales y domésticos, aquí elegimos concentrarnos en la dimensión del proceso productivo que se desarrolla en la ciudad de Córdoba. Es decir, antes de que la chicha sea tomada e incluso antes de que el maíz se convierta en chicha. El recorte surge como resultado del desarrollo de nuestro trabajo de campo, sobre el que consideramos necesario detenernos brevemente aquí.

Es preciso resaltar, en primera instancia, que nuestro primer acercamiento al universo de la chicha fue bibliográfico y con un «interés arqueológico», ya que se relacionaba con nuestro estudio de las actividades domésticas llevadas a cabo en una serie de sitios arqueológicos prehispánicos de la actual provincia de Catamarca ${ }^{2}$. Al interiorizarnos en las dimensiones simbólicas y sociales que la ingesta de esta bebida suponía en todo el mundo andino, consideramos necesario participar de fiestas y celebraciones en donde pudiéramos registrar y experimentar este tipo de consumo; así, a fines del año 2005, comenzamos a frecuentar las fiestas de la comunidad boliviana radicada en la ciudad de Córdoba. En esas ocasiones, y sosteniendo nuestra mirada «arqueológica», nos enfocamos en la cultura material que se encontraba involucrada en la producción y consumo de chicha. Comenzamos a preguntarnos cuáles eran las características específicas que este hacer y este beber adquirían en la ciudad de Córdoba, en un contexto de inmigración en el que los objetos (vasijas, por ejemplo) y materias primas a los que tradicionalmente se asociaba esta bebida no estaban presentes. Con este objetivo, continuamos participando de carnavales y festividades religiosas en donde se bebía chicha, conocimos y entrevistamos a las mujeres encargadas de su elaboración y venta, recorrimos aquellos espacios de la ciudad (ferias y mercados) relacionados con la comercialización de su materia prima esencial y también nos involucramos como ayudantes de un proceso de producción. De a poco, fuimos construyendo una representación del proceso que se llevaba a 
cabo en Córdoba, intentando reconocer los contextos materiales que ello suponía. Pero la imagen resultó ser mucho más compleja.

Hasta ese momento, habíamos reconocido en el proceso de producción de chicha a una instancia necesaria para poder contar con la bebida en ocasiones importantes para la comunidad (como fiestas, celebraciones o carnavales). La importancia que adquiría su consumo (como una instancia más de producción de lo local) hacía que su proceso de elaboración fuera visto en términos teleológicos. Sin embargo, los relatos de las chicheras comenzaron a mostrarnos las tensiones que permitían situar ese proceso de elaboración en el marco de las condiciones generales de marginalidad a las que están sometidos la mayoría de los inmigrantes, así como reconocer el espacio en el que se practicaban sus resistencias. Es decir, comprendimos que en la práctica de la elaboración de chicha se expresaba una política específica que se implicaba en la definición de los sujetos migrantes como pertenecientes a una comunidad, pero también era el lugar en donde se experimentaban las relaciones de marginalidad a las que se encontraban (y encuentran) sometidos en otros ámbitos. Comprendimos que beber chicha en Córdoba suponía un hacer muy conflictivo. Para profundizar sobre este punto consideraremos primero la situación de las chicheras en Córdoba, junto a sus prácticas de procuramiento de materias primas y de objetos, para luego concentrarnos en la manera en que todo ello define a un modo de hacer específico.

\section{Trayectorias conflictivas: chicheras, wiñapu, objetos}

La transformación del maíz en chicha supone un proceso que involucra a distintas personas, objetos y materias primas. En Córdoba, cada una de ellos cuenta con trayectorias complejas que se articulan con las condiciones que impone un contexto que es distinto (y adverso) al de sus lugares de origen. En este sentido, la imposibilidad o dificultad de abastecerse de las materias primas y objetos necesarios genera la puesta en acción de estrategias de abastecimiento y reemplazos que definen un modo particular de hacer, en el que las chicheras suelen ser su agente más visible.

\section{Las chicheras de Córdoba}

En la ciudad de Córdoba, la elaboración de chicha es una tarea principalmente femenina, en la que los hombres no se involucran sino como ayudantes (en la recolección de leña o en los procesos de batido, por ejemplo). Las chicheras no son muchas, de modo que sus saberes constituyen un capital escaso y valorado. Esta situación se traduce en una alta visibilidad de ellas dentro de la comunidad y de los barrios: en general, todos saben quién y cuándo hacen chicha (aunque no siempre cómo). No todas producen para las mismas fiestas, y en ciertos eventos públicos, en donde chicha y chicheras son protagonistas, los puestos de venta son rápidamente identificados.

Las chicheras a las que nos referimos son inmigrantes de origen boliviano (provienen de la ciudades de Cochabamba, Sucre y Potosí, y de un pueblo cercano a Cochabamba), con hasta diez años de residencia en la ciudad. Las condiciones económicas y materiales en las que se encuentran estas mujeres se inscriben en la situación de marginalidad y discriminación a la que se encuentran expuestos la mayoría de los inmigrantes. Viven en tres barrios periféricos de la ciudad de Córdoba, en algunos casos fuera de la denominada «Zona Urbana» donde los servicios públicos esenciales (tales como agua, luz, transporte) se encuentran ausentes. Gran parte de la comunidad reside en estos barrios, en ocasiones en situaciones de mucha pobreza. La migración se realiza principalmente en búsqueda de trabajo, por lo que las necesidades económicas obligan a tomar empleos mal pagos, en situaciones de extrema precariedad laboral y hasta de riesgo físico (como en los empleos en la construcción, para el caso de los hombres). En muchos hogares, incluso, el trabajo familiar en cortaderos de ladrillos o en huertas de zonas periféricas de la ciudad supone extensas jornadas laborales para todos sus miembros (ya que la paga es por cantidad producida y no por horas de trabajo), lo que obliga a que los más pequeños y jóvenes se alejen de ámbitos escolares y otros de socialización. Las asociaciones que nuclean a los miembros de la comunidad denuncian, desde hace años, a los empleadores que se aprovechan de su condición de inmigrantes y de sus necesidades económicas para generar relaciones laborales de explotación. Existe, además, un contexto de discriminación general en el que las manifestaciones culturales de la comunidad suelen ser objeto de rechazo y burla. Esta situación de marginalización es donde también se definen los conflictos de los procesos de elaboración de chicha.

En particular, las mujeres chicheras se dedican a la zapatería ambulante y al comercio de distintos productos en verdulerías, a través de la venta callejera y en ferias. Frecuentemente, este tipo de venta supone que las mujeres deban sufrir los embates represivos del municipio que intenta «limpiar» las zonas de la ciudad en donde se ubican los puestos de venta, tal como veremos más adelante. Muchas de ellas también se emplean como trabajadoras domésticas en 
barrios de clase alta, frecuentemente en situaciones de inseguridad laboral. Las jornadas de estas mujeres, por tanto, suelen ser muy largas y agotadoras, con lo que ninguna puede dedicarse tiempo completo a la elaboración de chicha: la producción se realiza para determinadas conmemoraciones y festejos, públicos y privados (celebraciones religiosas, carnavales y otras ocasiones importantes tales como casamientos o cumpleaños).

De todas maneras, y aun contando con el tiempo y los recursos necesarios, otras dificultades surgen del aprovisionamiento de las diferentes materialidades (materias primas y objetos) que se involucran en la elaboración. Ello se debe a que, si bien algunos «ingredientes» se consiguen y reemplazan fácilmente (como la chancaca -dulce de azúcar de caña), existe uno esencial e indispensable que es imposible de hallar en Córdoba: el wiñapu.

\section{No pasarás: el wiñapu y las fronteras}

El wiñapu es la materia prima esencial para hacer chicha de maíz. Se trata de una harina blanquecina realizada a base de maíz recién cosechado que es sometido a un proceso de malteado («hacer brotar») y luego molido. Se produce en Bolivia (y en el resto de los países andinos consumidores de chicha) y existen momentos del año en donde hay más wiñapu disponible que en otros. Si el objetivo es hacer chicha, lo primero será conseguir wiñapu. Y aunque las chicheras relatan que en Bolivia ellas mismas lo producían, todas coinciden en que el maíz cultivado en Argentina no es bueno para eso. En sus relatos se establece una clara diferencia entre el maíz que se consigue en sus lugares de origen y aquél que se compra en Córdoba:

... pero no se porqué este maíz [argentino] es duro... eso es... en cambio el maíz de nosotros es muy blando, blando... (Olga, 50 años).

Aguanoso es pues, cuando haces también de ese choclo, este el agua de... aguanoso es... con harina todavía tienes que mezclar y de allá no, pues espeso, pues no más es choclo también allá, rico es pues allá, aquí no, no me gusta, tal vez no he conocido comprando yo no (Aquilina, 38 años).

La primera frase corresponde a la entrevista de Olga, una mujer de 50 años, que se reconocía como no-chichera, mientras que la segunda a Aquilina, una chichera de 38 años. Es interesante notar, en el primer extracto de entrevista, la alusión a un nosotros, en oposición a un (tácito) ustedes y, en el segundo extracto, la alusión a un allá en oposición a un aquí. Ambos haciendo referencia al choclo o maíz boliviano, como más blando, espeso y rico. La única alternativa para hacer una bebida buena parece ser conseguir wiñapu producido en su lugar de origen y para eso es necesario "hacerlo traen».

Traer el wiñapu depende de algunos pocos comerciantes que se dedican a su distribución en Córdoba así como de las redes sociales con las que cuenten. De todas maneras, conseguir un buen producto en Bolivia y comprarlo no es un problema, ya que en general se trata de una harina de uso común. El conflicto se presenta en la aduana, porque el wiñapu no puede pasar por allí. La experiencia del tránsito por la frontera -y más específicamente por los pasos aduaneros- es relatada por las chicheras y vendedores como algo traumático e incluso puede constituirse en una causa por la cual se resigne la producción.

Un ejemplo puede ilustrar mejor. Hace casi dos años, tuvimos la intención de participar de un proceso de producción para el cual necesitaríamos wiñapu. En esa ocasión una de las personas que iba a implicarse en la producción (un boliviano residente en la ciudad de Córdoba desde hacía 30 años) y que estaba a punto de viajar a Bolivia, se ofreció para traernos unos pocos kilos. Sin embargo, como había experimentado en ocasiones anteriores la negativa de los inspectores al paso de productos de ese tipo, nos advirtió que su ingreso no sería sencillo y que quizá requeriría de nuestra ayuda desde Córdoba. Cuando llegó a Bolivia compró seis kilogramos de un muy buen wiñapu, que estaban envueltos en una bolsa plástica negra que serviría de protección durante el viaje, pero, tal como lo hubiera anunciado, fue detenido en un paso aduanero. Se le prohibió la entrada alegando que los inspectores debían evaluar primero si ese producto se trataba de algún tipo de sustancia ilegal. $Y$ aunque no pudieron demostrarlo, el paso le seguía prohibido. En ese momento, desde nuestro lugar hicimos el reclamo a través de una nota en la que se especificaba que el wiñapu que él traía formaría parte de un proceso de producción en conjunto con el ámbito universitario. El reclamo incluyó cartas, faxes y llamadas telefónicas. Finalmente, el wiñapu pasó. ¿Qué había sucedido?

El wiñapu es de origen vegetal y como tal se encontraría regido por las disposiciones ${ }^{3}$ específicas que regulan el tránsito de este tipo de productos. En ellas se señala que pueden ser ingresados por la frontera de forma libre y sin autorización previa aquellas materias primas que estuvieron sometidas a una serie de procesos, tales como malteado, machacado y molido (SENASA 1999). Casualmente, estos procesos son los que definen el carácter del wiñapu. Si de ello dependiera esta harina pasaría, pero el 
obstáculo aparece por su condición de «manufactura casera» y por las prohibiciones de paso que tienen todos los productos que se incluyen en estas categorías. Además, en el caso comentado, la harina poseía un envoltorio "dudoso y/o rudimentario», algo que es característico del perfil de riesgo de equipajes que los inspectores deben tener en cuenta al momento de realizar sus requisas (SENASA 1999). Es decir, la bolsa de plástico negro que envolvía el wiñapu definía otra frontera, otro límite que no podía ser cruzado: el de lo «casero» $y$ el de lo «dudoso».

En el transcurso de pocas horas, el wiñapu cambió de estado muchas veces, oscilando entre una "manufactura casera» de "envoltorio dudoso» con el paso prohibido, hasta llegar a convertirse en un «material de estudio» para la universidad. Sólo así pudo traspasar la frontera, dejando de ser un simple «producto vegetal».

El poder transformador de las palabras enunciadas por una institución académica argentina, y materializadas en una nota autorizadora, provocó este cambio de estado en el wiñapu, que dejó de ser aquél alimento «peligroso para el estatus fitozoosanitario argentino» y se convirtió en un objeto con «fines académicos». Al mismo tiempo, además, se relevaba a la aduana de la responsabilidad de su control y se comprometía al ámbito universitario a hacerlo. Pero no sólo el wiñapu cambió de estado; quien lo transportaba también fue transformado en un sujeto distinto, uno que se encontraba al mando de un "otro» universitario.

En otras palabras, en la situación que aquí comentamos, el wiñapu, tal como y como es concebido y significado por los inmigrantes, no pudo ser ingresado. A esa aduana llegó un sujeto boliviano (radicado en la ciudad de Córdoba desde hace más de 30 años y con DNI argentino) transportando wiñapu para chicha. Pero a esa aduana la cruzó un inmigrante mandadero de la universidad con un material de estudio bajo el brazo. Ambos habían cambiado.

Si volvemos a las palabras de las chicheras y comerciantes con relación al trásito por la aduana, nuevamente nos encontramos con los conflictos: «No nos dejan pasar nada», «Te lo quitan todo», "No te dejan nada». Las normativas que establecen limitaciones para el tránsito de productos «caseros», que también podríamos llamar domésticos, atentan contra las estrategias de reproducción cotidiana y contra formas de socialización primaria de un modo de vida que no es definido sino en términos de marginalidad. Dentro de ellos se incluyen, como la categoría más numerosa, los recursos alimenticios, sea en estado de materia prima o de productos acabados. Aquí también reconocemos una tensión, como la descripta más arriba, entre la necesidad de abastecerse de determinados productos y la dificultad de hacerlo; en ella se define la especificidad de los conflictos y negociaciones de estas transacciones, su gastro-política.

Volviendo a nuestro ejemplo, es necesario aclarar que nuestro pedido era por una cantidad pequeña de harina. Porque aún imaginando la posibilidad de que una harina de este tipo pudiera hacer su entrada al país, las normativas disponen que para aquellos productos destinados al consumo personal, sólo se pueden ingresar «pequeñas cantidades». Pero ¿cómo explicar que para hacer chicha se necesitan muchos kilos de wiñapu? Nuestro caso contemplaba sólo unos seis kilos, que luego se trasformaron en treinta litros de chicha. En términos "festivos», esa cantidad es insignificante y en caso de hacer traer wiñapu para la chicha de una fiesta deberíamos pensar, cómo mínimo, en unos 50 kilos. De hecho, en los carnavales que organiza la comunidad se registra la fabricación y venta de al menos 300 litros de chicha (sólo por dos chicheras), lo que implica aproximadamente unos 60 kilos de harina de wiñapu. Y si a eso agregamos que los carnavales duran varias semanas, y que por cada fin de semana se produce, vende y consume esta cantidad, deberíamos pensar en unos 250 kilos de wiñapu mensuales, sólo entre dos chicheras, considerando el mes más festivo y en un solo lugar de festejo.

No obstante, y más allá de las normativas y disposiciones, del carácter «casero» o de su envoltorio dudoso, el wiñapu pasa. Y lo hace por donde puede, incluso sorteando los controles y clasificaciones a los que puede ser sometido. Si nuestro acento está puesto en las materialidades que convergen en el hacer chicha quizá aquí nos encontramos con la más grande de ellas: es mucho wiñapu para una aduana que impide su paso.

Una vez en Córdoba, tampoco es simple encontrarlo ya que no son muchas las personas que se encargan de recibir y vender wiñapu. Su venta no parece ser esencial para la economía de ningún vendedor y no siempre es fácil dar con ellos. En particular, porque se tratan de puestos de venta que se ubican en ferias (las denominadas «ferias francas» que se establecen en lugares especiales y en determinados días de la semana) o a la vera de grandes mercados de la ciudad. En ambos casos, pero sobre todo en el segundo, la materialidad de los puestos evoca las prácticas de comercialización en Bolivia. Se encuentran regenteados por mujeres y a veces sólo compuestos por awayos (manta rectangular, tejida tradicionalmente en telar, utilizado para la carga de niños u objetos), o por cajones, mesas y balanzas, lo que los hace móviles y fácilmente transportables. Esta 
movilidad aumenta, además, porque las vendedoras deben sortear las embestidas regulares de inspectores que intentan desbaratar sus puestos (de frutas y verduras, generalmente) bajo el argumento de la falta de habilitación, a lo que se suma su condición de mujeres e inmigrantes.

Estas embestidas han sido denunciadas durante años por las asociaciones que congregan a los miembros de la comunidad. Durante el año 2007 en particular, y bajo la consigna de lograr un mercado más «limpio» y ordenado, el gobierno de la ciudad dispuso acciones represivas contra estas mujeres y sus puestos. Bajo estas consignas, se penalizan las prácticas usuales de venta y presentación de productos de las mujeres inmigrantes generando un límite material entre el mercado legal de la ciudad (y su edificio) y los puestos de la periferia (con sus awayos en el piso). Se construye una nueva frontera para aquello que puede ser vendido, comprado y, en última instancia, comido y bebido.

El wiñapu encuentra en estos puestos el final de un recorrido que comenzó en el lugar en el que fue comprado; un recorrido en el que él mismo fue definiéndose como materia prima para chicha, pero también como producto con el paso prohibido y como integrante de un puesto de venta perseguido. Ya no es el mismo producto: en torno a él se han construido estrategias de adquisición, se han desarrollado negociaciones y enfrentamientos. Todo ello en un nuevo contexto en el que el wiñapu ha sido el objeto de la disputa y en el que se definen las situaciones de marginalidad y discriminación de los inmigrantes.

No obstante, otras materialidades se implican en la elaboración de chicha y suponen la puesta en juego de otras estrategias en este mismo contexto: nos referimos a los objetos que suplantarán a aquellos utilizados en los lugares de origen de las chicheras.

\section{Los objetos involucrados en la producción}

En los lugares de origen de las chicheras, la chicha y su proceso de producción se asocia de manera directa, y en algunos casos unívoca, con tres objetos en particular: virkes, puñus y peroles (los dos primeros de cerámica y el último de metal), a los que luego se suman las cucharas de madera para remover y otras vasijas o recipientes pequeños que se utilizan durante el traspaso de líquidos. Uno de ellos son las tutumas (recipientes fabricados a partir de calabazas o porongos), que también se utilizan para el momento del consumo.

La provisión de estos objetos específicos en Córdoba no siempre es posible y por lo tanto suelen ser reemplazados por otros que poseen algunas características morfológicas compartidas y que pueden cumplir funciones similares: baldes de plástico, grandes ollas de metal y «tachos de aceite» (de 200 litros). Sin embargo, la elección de los reemplazos en Córdoba no deja de evocar nunca a los objetos de Bolivia, muchos de los cuales fueron dejados bajo la custodia de familiares al momento de la migración: «Todos están, pues, allá... todos... todos... mi mamá debe estar usando esos puñus» (Aquilina, chichera, 38 años). En cierto sentido, el hecho de que en Bolivia los objetos sigan utilizándose es lo que permite que los baldes de plástico en Córdoba puedan ser usados para las mismas funciones que aquellos; es decir, en la práctica de elaborar chicha en Córdoba, el significado de los objetos se construye a partir de los sentidos que se les otorgan en Bolivia.

Las referencias hacia los objetos de cerámica (es decir, aquellos que se utilizan en los lugares de origen de las chicheras) siempre se asocian con las maneras en que deben ser cuidados y conservados durante el proceso de producción y durante su posterior lavado, lo que les confiere a cada uno de ellos trayectorias particulares. Con relación al puñu, una chichera relataba:

Mi mamá le cuida harto, como huevito le hace... (risas) ni los hombres manejan, como un huevito así, le hace manejar, no, no, con calmita (...)

Por eso cuando yo me voy a morir, tu te lo vas a agarrar y así te lo vas a cuidar, me dice a mí... en quechua me dice, así. Como yo también me cuido, así también tu tienes que cuidarte, hijita, yo te lo voy a dejar cuando me muera. (Aquilina, chichera, 38 años).

En cambio, en torno a los objetos de plástico utilizados en Córdoba, las referencias en relación a su cuidado y limpieza no abundan, ya que no les pasa nada y por otro lado, son fácilmente reemplazables (con excepción del tacho usado para la fermentación).

De los objetos mencionados, los virkes son vasijas de cerámica de dimensiones variables, con grandes bocas que se utilizan en el mezclado y batido de los ingredientes en distintos momentos del proceso; en Córdoba se reemplazan por baldes y fuentones de plástico. Los peroles son recipientes de metal (ollas con boca ancha de diferentes tamaños) que se utilizan principalmente durante la etapa de cocción, que suele ser una de las más prolongadas del proceso; en general, son los más fáciles de reemplazar, y se lo hace por grandes ollas metálicas. En el caso de los puñus, también son vasijas cerámicas de grandes dimensiones, con una base cóncava y boca 
pequeña que intervienen en una única y fundamental tarea: la fermentación final de la bebida. Aquí se reemplazan por tachos de aceite, con una capacidad promedio de 200 litros, aunque en casos en donde la producción no es muy grande pueden utilizarse otros recipientes, como un conjunto de tachos de pintura (de 20 litros de capacidad cada uno). Luego de la fermentación no suele existir ningún proceso de almacenamiento ni objeto destinado a tal fin, la chicha es tomada inmediatamente: ¿para qué vas a guardar vos?, ...hay que tomar, compartir.

La provisión de objetos tiene diferentes fuentes. En todos los casos, las chicheras son dueñas de los grandes tachos de plástico que se utilizan en la fermentación; su posesión es muy importante, ya que cuando se produce para vender o para una gran celebración, es necesario hacerlo en grandes cantidades. Sin embargo, proveerse de estos tachos no es algo sencillo y requiere de un seguimiento de la vida útil de los mismos en sus contextos de uso primario (establecimientos de venta de combustibles, por ejemplo) hasta el momento de su descarte, cuando ya es posible adquirirlo o comprarlo. Al igual que los puñus de cerámica que sólo se usan para fermentar la chicha, los tachos de aceite también se utilizan sólo para eso.

En el caso de los baldes, fuentones y ollas para la cocción, todos ellos de uso cotidiano, la chichera utiliza los de su propiedad así como también puede pedirlos en préstamo a vecinos de la misma comunidad. El préstamo de objetos, así como de mano de obra durante la producción, involucra un compromiso de reciprocidad que supone la entrega de cierta parte de la producción de chicha, junto con la devolución del objeto. En el caso de las tutumas pueden ser fabricadas con calabazas en Córdoba, pero como son recipientes pequeños hay muchos de ellos que pudieron ser traídos desde los lugares de origen. Su boca ancha es útil durante la acción de separar los líquidos que han decantado en el proceso de elaboración, por lo que su reemplazo por vasos o tazas, aunque se hace necesario, no es del todo eficiente: "para sacar... de un lado a otro, para sacar los líquidos, la tutuma... es de planta que se llama «bolo» allá, y acá le Ilamamos porongo, pero allá son más grande» (Aquilina). Una de las chicheras fabrica sus propias tutumas con porongos que se consiguen en Córdoba.

La leña que se utiliza para la cocción (que dura muchas horas) tiene diversas fuentes. Puede ser recolectada de las inmediaciones de los barrios, ya que muchos de ellos se encuentran en los límites de la ciudad (uno fuera del ejido urbano) y colindando con áreas con la presencia de pequeños árboles. En esta tarea se involucran principalmente los hombres, aunque también participan las mujeres y los niños. En uno de los casos, registramos el uso de listones de madera que habían sido descartados de una obra en construcción en la que se empleaba como peón el marido de una chichera. Nuevamente, la falta de tiempo y de recursos económicos condiciona las posibilidades de acceso a la leña y obliga a adoptar distintas estrategias de abastecimiento. No hemos registrado situaciones de compra.

Por último, en los casos en que el destino de la chicha es la comercialización es necesario contar con muchos recipientes para su venta, en general botellas plásticas de uno o dos litros. Si bien algunos compradores suelen llevar su propio recipiente, frecuentemente es la chichera quien la proporciona. Pueden conseguirse por intermedio de vecinos y amigos o, como en una de las situaciones registradas, ser recolectadas por niños de los depósitos de basura, para luego lavarlas y presentarlas para la venta.

Como podemos apreciar, muchos de los objetos mencionados aquí son de uso cotidiano y se relacionan con actividades domésticas diferentes al mismo tiempo en que se involucran con determinados pasos o etapas del proceso de producción de chicha. Cada uno cuenta, además, con trayectorias particulares que convergen en este hacer, y que se encuentran definidas por la puesta en práctica de estrategias específicas de abastecimiento. Cabe resaltar, con respecto al ejemplo de la obtención de leña, que el lugar (la obra en construcción) de donde se obtuvo la madera en una ocasión sea el mismo en donde normalmente se viven las situaciones de precariedad e inseguridad laboral que definen la situación de marginalidad de los inmigrantes.

Estas trayectorias confluyen en los procesos de elaboración de chicha, momento durante el cual una «receta» se pone en práctica y se construye un nuevo sentido en cada uno de estos objetos. Se establecen distintas relaciones, no sólo con las actividades domésticas de las casas en Córdoba, sino también con los espacios domésticos de las casas en Bolivia. En este sentido, el hacer chicha es un proceso que involucra y produce diversidad de escalas, espaciales y temporales. En ellas se relacionan espacios y objetos distanciados físicamente, junto con recetas tradicionales y modos de hacer contemporáneos. Allí, el significado de los tachos, de los baldes de plástico y de las botellas de la basura, se construye a partir de la evocación constante de aquellos otros objetos (lejanos) a los que reemplazan así como de la receta que se hace presente. En este sentido, los contextos materiales que se producen en Córdoba definen este modo particular de hacer a través de un proceso iterativo, que evoca al que se lleva a cabo en Bolivia pero que también se distingue de él.

\section{El modo de hacer chicha en Córdoba}

Pero ¿cómo es que se define este modo particular de hacer? La chicha consta de un proceso 
de producción que implica mucho tiempo y diversidad de tareas (Cutler y Cárdenas 1947; Jennings et al 2005; Pazzarelli y Vargas 2007), por lo que en la ciudad de Córdoba las chicheras recurren generalmente a los fines de semana o días de descanso para llevarlos a cabo. Los espacios físicos utilizados suelen ser los patios de las casas, en donde es posible encender el fuego que se mantendrá durante muchas horas. Antes de comenzar, es necesario que cada uno de las materias primas, objetos y sujetos implicados en la producción se encuentren en el lugar, por lo que en ese momento confluyen por primera vez todas las trayectorias que hemos descripto anteriormente. Todas ellas se funden, entonces, en la definición de un modo específico de hacer chicha, en donde la chichera y el wiñapu boliviano se constituyen en los primeros protagonistas y en donde los objetos también hacen. Repasemos este modo de hacer.

El primero de los pasos es realizar una mezcla del wiñapu con agua hirviendo (puede agregársele chancaca-dulce de azúcar de caña-) hasta formar una masa donde las manos de las chicheras se hunden, retorciéndola de forma constante. Esa misma mezcla será depositada en el interior de los baldes que aguardan en el patio de la casa (y que suplantan a los virkes). Una vez allí se le agrega más agua hirviendo, hasta llenar el recipiente y se comienza a batir (dependiendo de la cantidad, durante una hora o dos) con una cuchara de madera hasta lograr una mezcla líquida homogénea de color oscuro. Durante este proceso la cuchara va pasando de mano en mano, entre todos aquellos (incluso hombres) que unos días después se juntarán nuevamente a tomar. En ese momento, la conversación puede fluir por muchos lugares, pero la chichera no descuidará el proceso: se inclinará frente a las bocas anchas de los baldes en donde los ingredientes se mezclan, mirando detenidamente el líquido que se mueve y tratando de encontrar leves cambios de tono en el color para reconocer si ya está llegando a su punto. A veces, hundirá los dedos en la mezcla durante un instante para luego sacarlos y palpar el líquido que quede entre ellos: no es necesario llevarlo a la boca, se prueba con los dedos.

Cuando está listo, se lo deja reposar para lograr que esta mezcla se separe en tres fases (dos líquidas, con diferentes densidades, y una sólida) que luego serán divididas. Con ayuda de una tutuma (o de un vaso) se traspasa la primera fase líquida de color oscuro llamada upi a otro recipiente. Luego se realiza el mismo procedimiento con la segunda fase líquida, denominada kheta, de color blanquecino y de mayor densidad que la primera. El sólido restante, que queda en el fondo del virke o balde, se denomina hanchi y, en general se lo descarta, aunque también puede utilizarse para hacer arrope o alimentar a los animales. La acción de separar el upi y la kheta supone manos firmes, decididas y pacientes que puedan reconocer el momento adecuado en el que los líquidos, entre traspaso y traspaso, vuelven a reposar y a separarse. La chichera evita los movimientos apresurados y se concentra, primero en el upi, luego en la kheta. En este proceso, la boca ancha de la tutuma permite que la acción pueda ser medida y controlada.

Una vez realizada esta separación, el upi queda reposando y enfriándose, mientras la kheta se coloca en un perol (u olla de metal) sobre el fuego para que comience su transformación. El objetivo es que se reduzca y adquiera una consistencia de melaza color marrón oscuro, proceso que puede demorar unas tres o cuatro horas como mínimo y durante el cual es necesario remover el líquido y atizar el fuego de manera constante. Ahora, la cuchara vuelve a pasar de mano en mano, entre la chichera y los ayudantes, mientras se remueve sin parar: «... de eso solito tiene que cocer, pero no tienes que aguantar ni agua, ni nada... no tienes que aguantar a la kheta... moviendo, moviendo, nomás tienes que hacer cocer» (Aquilina). El proceso termina cuando la cuchara puede ser colocada por la chichera en el centro de la olla y ser sostenida por la melaza espesa en la que se ha transformado la kheta; en palabras de la chichera: «que se quede sola». Cuando se llega a ese momento, después de muchas horas y cuando el sol ha comenzado a bajar (o a subir), el fuego deja de ser alimentado y todos, la chichera, los ayudantes, y la kheta reposan y descansan; y mientras la olla se enfría, comienza la espera para realizar la mezcla final.

Así, la kheta y el upi que estaba reposando serán mezclados juntos en un puñu (o tacho elegido para la fermentación) y dejados ahí durante una semana, aproximadamente. Para esto es necesario tapar la pequeña boca del recipiente con alguna manta, bolsa o con barro amasado: "Se va a un tacho, pues. A un tacho tienes que poner, $y$ en el tacho tienes que hacer macerar, pues, la chicha... con la kheta, con la upi, todo le mezclas y lo pones biencito, y ahí ya macera, pues, para chicha, ya...» (Aquilina). Luego de transcurrido ese período, la chicha estará lista para ser tomada.

Hasta este momento los tachos parecen no ingresar a la escena, pero en realidad han sido parte esencial desde el comienzo: han sido observados y observadores de un proceso del que recibirán aquello que debe ser resguardado. Durante esos días, no será posible tocar ni ver su contenido y deberán quedarse inmóviles, reposando en lugares retirados del paso cotidiano, en alguna zona oscura de la casa, acompañando silenciosamente el ritmo de las tareas domésticas. Hasta que la chicha esté lista; entonces terminará el silencio. La fermentación, 
ese proceso que parece no involucrar ya a las personas se define, en última instancia, por la comunicación con ellas. La materialidad del tacho y de las bolsas que sellan su boca, el volumen de bebida que allí reposa y las personas que circulan con atención a su alrededor son protagonistas de un mensaje que será enunciado y oído: cuando la chicha está llegando a su punto, las burbujas retumban contra las paredes del tacho y avisan que ya está lista para tomar. La chicha te llama.

Desde el comienzo hasta el final del proceso de elaboración, los patios de las casas de Córdoba reciben el wiñapu traído desde Bolivia para ser transformado en los baldes y tachos locales. Cada uno de los objetos que se involucran en la producción cuenta con una trayectoria distinta, pero que se enmarca en un contexto de inmigración en el que la provisión de muchos de ellos no es sencilla. El wiñapu ha sido objeto de disputa en distintos contextos, los tachos se han procurado luego poner en juego estrategias de aprovisionamiento cuando éstos fueron descartados de su función primaria y a través de los baldes y fuentones plásticos se han establecido compromisos de reciprocidad con otros paisanos. Hasta el tiempo necesario para ejecutar este proceso tuvo que ser «negociado» y restado de los días de descanso de las largas jornadas laborales de las chicheras.

En cada uno de los pasos reseñados, la chichera expone y actualiza su saber-hacer y establece los espacios a ocupar por cada uno de los ayudantes que serán luego consumidores. Se produce una poética de la elaboración de chicha a través de la cual se define el lugar de la chichera y de la bebida en un contexto que es distinto al del lugar de origen. Un contexto en el que se expresan las mismas relaciones de marginalidad a las que se resiste.

Como mencionamos anteriormente, el proceso de elaboración que se lleva a cabo en Córdoba evoca a aquél que se realiza en Bolivia, actualizándolo en el marco del contexto migratorio que impone duras condiciones de vida. En la descripción anterior de las etapas de la elaboración, la intención de considerar lo que los objetos hacen se constituye en un recurso para comenzar a pensar cómo con un determinado repertorio de objetos y de contextos materiales distintos al «original» la chicha se hace igual.

Lo que sucede en el patio de una casa boliviana en Córdoba cuando se elabora chicha excede el ámbito de la receta; se constituye, en cambio, en un escenario donde la política de la bebida se hace presente como técnica para la producción de lo local y como posibilidad de resistencia.

\section{Consideraciones finales}

Hacer chicha en la ciudad de Córdoba es un hecho en el que se involucran distintos objetos y sujetos y en donde se expresan y dirimen diversidad de conflictos. Hacer chicha supone enfrentar a una frontera y a una aduana que impide el paso del wiñapu así como soportar la interpelación estatal a través de inspectores que incautan una harina que en Bolivia es de uso cotidiano. Hacer chicha supone resistir los embates de las normativas municipales que prohíben una forma de exhibición y venta ambulante de productos, así como generar estrategias para conseguir los objetos adecuados para el proceso de elaboración. Cada una de estas instancias, a lo que se suman las condiciones económicas y laborales que sufren las chicheras, definen lo que significa hacer chicha en Córdoba. Por eso, el consumo que se produce en las fiestas y celebraciones se encuentra fuertemente ligado a su instancia de elaboración, en donde convergen las trayectorias de cada uno de los agentes que participaron de ella, sus conflictos y sus resistencias.

Reconocer las trayectorias de las mujeres chicheras, del wiñapu y de los objetos que se involucran en la producción de chicha es una manera de visibilizar los mecanismos que se encuentran dificultando de manera continua la posibilidad de un proceso de estas características, fuertemente significado por la comunidad. Un recorrido de este tipo, además, nos ha permitido registrar los contextos materiales en los que se expresan y producen las embestidas que soportan estos colectivos, y en los cuales se despliegan sus estrategias de resistencia. Los procesos de producción de chicha en la ciudad de Córdoba son instancias en las cuales se experimentan las mismas relaciones de subalternidad y marginalidad a las cuales se resiste; la producción de lo local se inscribe en este contexto.

Se trata de un hacer en el que se hace mucho más que una bebida: wiñapu, chicha, mujeres, hombres, objetos, espacios y tiempos se constituyen mutuamente en un proceso recursivo en donde todos confluyen. En esta práctica se produce una poética que actualiza los saberes de las chicheras en un contexto de migración y que construye lazos de compromiso y reciprocidad entre los sujetos de la comunidad. Pero también es el lugar en donde opera una gastro-política específica definida por las negociaciones y enfrentamientos con distintos tipos de agentes represivos que regulan el intercambio de materias primas y alimentos.

Es verdad que el proceso de elaboración de chicha en Córdoba se inscribe dentro de las condiciones de discriminación y subalternidad a las que están expuestos los inmigrantes, que son mucho más amplias que aquellas expresadas en este contexto específico. En este sentido, la gastro-política a la que aludimos no es sino una parte de una política de resistencia mucho más 
compleja que se despliega con motivo de las situaciones de precariedad laboral y económica de los migrantes y en el marco de la marginalización de muchas de sus otras prácticas alimenticias y culturales. Hacer visible parte de estas resistencias es, para nosotros, una forma de actualizar los reclamos de estos colectivos.

Córdoba, 8 de septiembre de 2008

\section{Agradecimientos}

Pensar en este trabajo no hubiera sido posible sin las mujeres chicheras que nos dejaron hacery beber chicha y compartieron con nosotros parte de sus días. Agradecemos también a los comentarios vertidos por un revisor que contribuyeron significativamente a mejorar la exposición de este trabajo.

\section{Bibliografía}

Abercrombie, T. 1993. Caminos de la memoria en un cosmos colonizado. Poética de la bebida y la conciencia histórica en K'ulta. Saignes, T. (comp.): Borrachera y memoria. La experiencia de los sagrado en los Andes, 139-170. Hisbol/IFEA, La Paz.

Appadurai, A. 1981. Gastro-politics in South Asia. American Ethnologist 8, 3:494-511.

2000 [1995] La construcción de lo local. La modernidad desbordada. Dimensiones culturales de la globalización. Capítulo 9: 187-207. Trilce FCE.

Arnold, D. (coord). 1998. Hacia un orden andino de las cosas. Tres pistas de los Andes meridionales. Hisbol/ILCA, Bolivia.

Bourdieu, P. 1977. Outline of a Theory of Practice. Cambridge Unversity Press, Cambridge.

Bourdieu, P. y L. Wacquant. 1995. Respuestas. Por una antropología reflexiva. Grijalbo, México.

Cutler, H. y M. Cárdenas. 1985 [1947]. Chicha, una cerveza indígena sudamericana. Lechtmann, $\mathrm{H}$; Soldi, A. M. (coord.): La tecnología en el mundo andino, 247-259. UNAM, México.

Fernández Juárez, G. 1997. Entre la repugnancia y la seducción. Ofrendas complejas en los Andes del Sur. Centro de estudios regionales andinos, Perú.

Giddens, A. 1984. The Constitution of Society. Outline of the Theory of Structuration. University of California Press, Berkeley y Los Ángeles.
Hodder, I. 1990. The Domestication of Europe. Structure and Contingency in Neolithic Societies. Basil Blackwell, Oxford.

Jennings, J., K. Antrobus, S. Atencio, E. Glavich, R. Johnson, G. Loffler, y C. Luu. 2005. 'Drinking Beer in a Blissful Mood' Alcohol Production, Operational Chains, and Feasting in the Ancient World. Current Anthropology 46, 2:275:304.

Paredes, M. R. 1936. Mitos, supersticiones y supervivencias populares de Bolivia. Imprenta Atenea, La Paz, Bolivia.

Pazzarelli, F. y G. Vargas Ibarra. 2007. Rico es pues allá. Chicheras, recetas y objetos en un proceso de producción de chicha en Córdoba. Actas, IV Reunión Internacional de Teoría Arqueológica en América del Sur / Inter-Congreso del WAC, 180181. Catamarca.

Randall, R. 1993. Los dos vasos. Cosmovisión y política de la embriaguez desde el incanato hasta la colonia. Saignes, T (comp.), Borrachera y memoria. La experiencia de los sagrado en los Andes. 73:1 12. Hisbol/IFEA, La Paz

Saignes, T. 1993. «Estar en otra cabeza»: tomar en los Andes. Saignes, T (comp.), Borrachera y memoria. La experiencia de los sagrado en los Andes. 11:21. Hisbol/IFEA, La Paz.

SENASA. 1999. Manual de procedimientos en control de personas, equipajes y vehículos de transporte en puestos de frontera. Resolución $\mathrm{N}^{\circ}$ 295/99. Link http//www.senasa.gov.ar. Último acceso $2 / 07 / 08$.

Shanks, M. y C. Tilley. 1987. Re-constructing archaeology. Theory and practice. Cambridge University Press, Cambridge.

Rostworoski, M. 2004. Costa Peruana Prehispánica. Instituto de Estudios Peruanos, Lima.

' Con respecto a los encargados de producir chicha, algunos autores como Rostworowski (2004) proponen que para la costa peruana los hombres son los chicheros, mientras que en la región de la sierra son las mujeres. En la situación que aquí describimos, las mujeres son las que realizan casi la totalidad de las tareas que supone producir chicha.

${ }^{2}$ Se tratan de los sitios arqueológicos ubicados en el Valle de Ambato, provincia de Catamarca, que fueron ocupados entre los siglos VI y XII de nuestra era, por la denominada cultura de «La Aguada«. El Proyecto Arqueológico Ambato se desarrolla en el Museo de Antropología (FFyH, UNC) bajo la dirección general del Dr. Andrés Laguens. En la actualidad, uno de los autores desarrolla su investigación sobre la cultura material asociada a las prácticas de alimentación en Ambato, 
entre las que se cuenta la posible elaboración de chicha en grandes vasijas cerámicas.

${ }^{3}$ Estas disposiciones son dictadas por el SENASA (Servicio Nacional de Sanidad y Calidad Agroalimentaria), «el organismo del Estado argentino encargado de ejecutar las políticas nacionales en materia de sanidad y calidad animal y vegetal ...» y que comprende, entre otras tareas, «el control del tráfico federal, importaciones y exportaciones de los productos, subproductos y derivados de origen animal y vegetal, productos agroalimentarios, fármacoveterinarios y agroquímicos, fertilizantes y enmiendas.» (www.senasa.gov.ar) 